\title{
Tisotumab Vedotin in Cervical Cancer: Current Status and Future Perspectives
}

\author{
Elena Giudice, ${ }^{1}$ Floriana Camarda, ${ }^{2}$ Vanda Salutari, ${ }^{3}$ Caterina Ricci, ${ }^{3}$ Camilla Nero, ${ }^{3}$ Maria Vittoria Carbone ${ }^{3}$ Viola Ghizzoni, \\ Lucia Musacchio, ${ }^{3}$ Chiara Landolfo, ${ }^{3}$ Maria Teresa Perri, ${ }^{1}$ Giovanni Scambia ${ }^{1,3}$ and Domenica Lorusso ${ }^{1,3}$ \\ 1. Institute of Obstetrics and Gynecology, Università Cattolica del Sacro Cuore, Fondazione Policlinico Universitario A. Gemelli IRCCS, Rome, Italy; \\ 2. Oncologia Medica, Comprehensive Cancer Center, Università Cattolica del Sacro Cuore, Fondazione Policlinico Agostino Gemelli IRCCS, Rome, \\ Italy; 3. Department of Woman, Child and Public Health, Fondazione Policlinico Universitario A. Gemelli IRCCS, Rome, Italy
}

DOI: https://doi.org/10.17925/OHR.2021.17.2.68

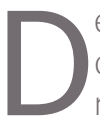
espite the introduction of screening programmes and vaccination campaigns, cervical cancer (CC) still represents one of the leading causes of cancer-related mortality in women worldwide and the fourth most common female malignancy. The prognosis of CC remains poor, especially for late-stage and relapsed disease. While platinum-based chemotherapy with or without bevacizumab has been the established standard of care in the first-line setting, no standard second-line treatments have been identified, and new active agents are needed. Antibody-drug conjugates (ADCs) represent a novel class of agents approved by the US Food and Drug Administration to treat haematological malignancies and metastatic breast cancer. According to available data, ADCs could also represent a valid option for metastatic and recurrent CC. This review describes the role of tisotumab vedotin, an ADC targeting tissue factor, in the metastatic and recurrent $\mathrm{CC}$ setting. An overview of published data and ongoing clinical trials is also provided.

\section{Keywords}

Cervical cancer, chemotherapy, antibody-drug conjugate, tisotumab vedotin, clinical trials

Disclosure: Vanda Salutari has received grants from Clovis Oncology, Tesaro, GSK, AstraZeneca, MSD, PharmaMar, Eisai and Roche, outside the submitted work. Giovanni Scambia has received personal fees from Roche, Clovis Oncology, AstraZeneca, PharmaMar and Tesaro, outside the submitted work. Domenica Lorusso has received grants from Roche, Incyte and Immunogen, grants and personal fees from GSK, Clovis Oncology, MSD, PharmaMar, Genmab and AstraZeneca, and personal fees from Amgen outside the submitted work. Elena Giudice, Floriana Camarda, Caterina Ricci, Camilla Nero, Maria Vittoria Carbone, Viola Ghizzoni, Lucia Musacchio, Chiara Landolfo and Maria Teresa Perri have no financial or non-financial relationships or activities to declare in relation to this article.

Review process: Double-blind peer review.

Compliance with ethics: This study involves a review of the literature and did not involve any studies with human or animal subjects performed by any of the authors.

Data availability: Data sharing is not applicable to this article as no datasets were generated or analysed during the current study.

Authorship: The named authors meet the Internationa Committee of Medical Journal Editors (ICMJE) criteria for authorship of this manuscript, take responsibility for the integrity of the work as a whole and have given final approval for the version to be published.

Access: This article is freely accessible at touchONCOLOGY.com (C) Touch Medical Media 2021

Received: 5 August 2021

Accepted: 30 September 2021

Published online: 29 November 2021

Citation: touchREVIEWS in Oncology \& Haematology. 2021;17(2):68-71

Corresponding author: Domenica Lorusso, Department of Woman, Child and Public Health, Fondazione Policlinico Universitario A. Gemell IRCCS, Largo Agostino Gemelli 8, 00168, Rome Italy.E: domenica.lorusso@policlinicogemelli.t

Support: No funding was received in the publication of this article.
Cervical cancer (CC) is the fourth most common female malignancy, with 640,127 new cases and 341,831 deaths in 2020 worldwide. Despite the introduction of screening programmes and vaccination campaigns, CC still represents one of the leading causes of cancer-related death in women. ${ }^{1}$

The appropriate treatment strategy is determined according to the stage of disease at diagnosis Patients with early-stage and locally advanced disease are treated with potentially curative intent with surgery and exclusive concomitant chemo-radiotherapy, respectively. In contrast, patients with metastatic, recurrent and persistent disease have a dismal prognosis, with a 5-year survival rate of less than $20 \%{ }^{2}$ In these patients, platinum-based chemotherapy represents the standard of care. The Gynecologic Oncology Group protocol 240 (GOG-240) trial reported a significant improvement in overall survival (OS) in patients treated with chemotherapy plus bevacizumab compared with chemotherapy alone (16.8 months versus 13.3 months, hazard ratio $0.77,95 \%$ confidence interval [Cl] 0.62-0.95; $\mathrm{p}=0.0068){ }^{3.4}$ Unfortunately, effective second-line treatments are lacking for patients who progress on first-line chemotherapy. Several cytotoxic agents, including vinorelbine, topotecan, gemcitabine and pemetrexed, have been tested, with low reported response rates ranging between $4.5 \%$ and $20 \%$, as well as poor median OS of about 5 months. ${ }^{5}$ In this scenario, the evaluation of new, more active treatment strategies represents an open field of research and an urgent unmet clinical need.

In recent years, clinical studies have focused on the possible role of immunotherapy in recurrent or metastatic CC, and in 2018, the US Food and Drug Administration (FDA) approved pembrolizumab based on the KEYNOTE-158 study ${ }^{6}$ for advanced/recurrent CC expressing programmed cell death-1.

\section{A new perspective: Antibody-drug conjugates}

Antibody-drug conjugates (ADCs) are a novel class of agents consisting of three main components: antibody, linker and payload. 'The monoclonal antibody interacts with an antigen expressed on tumour cells, and with the help of the linker, cytotoxic agents are conveyed inside the cell and degraded by Iysosomes. Once free, the cytotoxic effector can perform its function, causing cell death through several mechanisms. The monoclonal antibody recognizes an antigen predominantly expressed on tumour cells relative to normal cells in order to maximize efficacy while reducing toxicity. The monoclonal antibodies are mainly represented by the immunoglobin (IgG) subtype engineered to join payload molecules. A stable attachment is granted by cysteine variations or mutations that are able to modify electric charge and create stable bonds between immunoglobulins and cytotoxic agents The drug:antibody ratio is defined as the number of payloads attached to IgG; however, a higher drug:antibody ratio does not necessarily confer enhanced efficacy because it could interfere with 
the pharmacokinetics of the ADC. ${ }^{.}$The antigens targeted in gynaecological cancers include folate receptor $\alpha$, sodium-dependent phosphate transport protein 2B, tissue factor (TF), mesothelin and mucin 16.

The payload is usually represented by small cytotoxic molecules measuring 300-1,000 daltons; agents with significant efficacy are auristatin and maytansine, both potent microtubule inhibitors. ${ }^{9}$ Among auristatins, monomethyl auristatin E (MMAE/vedotin) has shown a bystander effect on neighbouring cells owing to its ability to cross cell membranes. Monomethyl auristatin F (MMAF/mafodotin), however, did not show the same activity. Among maytansines, we distinguish emtansine, mertansine, soravtansine and ravtansine.

The linker is fundamental to the stability of ADCs in the bloodstream, avoiding any inappropriate release with subsequent major adverse effects. The linkage between the main components can be cleavable or non-cleavable. Cleavable linkers are split by enzymatic degradation and hydrolysis reactions, whereas non-cleavable linkers are divided within lysosomes, together with the antibody backbone.

The first-in-class ADCs were approved by the FDA for the treatment of haematological malignancies: brentuximab vedotin for Hodgkin lymphoma and anaplastic large cell lymphoma, gemtuzumab ozogamicin for acute myeloid leukaemia, and inotuzumab ozogamicin for B-cell acute lymphoblastic leukaemia. ${ }^{10}$ Concerning solid tumours, only adotrastuzumab emtansine is approved for the treatment of advanced human epidermal growth factor receptor 2-positive breast cancer. ${ }^{11}$

Among gynaecological malignancies, several clinical trials have investigated the role of ADCs. The activity of mirvetuximab soravtansine has been explored in ovarian cancer and endometrial cancer, while anetumab ravtansine (DMOT4039A) and sofituzumab vedotin (DMUC5754A) have both been studied in ovarian cancer. The efficacy and safety of tisotumab vedotin (TV) have been investigated in ovarian, endometrial and cervical cancers. The aim of the current review is to describe the role of TV in metastatic and recurrent $\mathrm{CC}$, and to provide an overview of published and ongoing clinical trials in this setting.

\section{Methods}

Following the Preferred Reporting Items for Systematic Reviews and Meta-Analyses (PRISMA) guidelines, a search of PubMed and Scopus databases was performed to identify publications up to 25 April 2021. The following combination of Medical Subject Headings (MeSH) terms was used in both databases: "cervix cancer", "cervical cancer", "solid tumor", "tisotumab vedotin", "antibody-drug conjugated". We included published clinical trials without limitations on the publication date. We also searched ClinicalTrials.gov for ongoing trials. We excluded reviews, abstracts and articles not written in English.

\section{Tisotumab vedotin: An overview}

TV is an ADC targeting TF, a transmembrane glycoprotein mainly known as the primary initiator of the extrinsic coagulation pathway. ${ }^{12}$

TF is physiologically expressed on subendothelial cells (fibroblasts, pericytes and smooth muscle cells), and the exposition, secondary to injury, promotes the activation of blood-circulating factor VII, leading to coagulation. ${ }^{13}$ Moreover, the TF-factor VIla complex mediates an intracellular signalling cascade by activating protease-activated receptor 2 , leading to the production of pro-angiogenetic factors and cytokines. ${ }^{12}$ Alongside its function as a coagulation promoter, TF also plays a role in oncogenesis, promoting tumour cell survival, tumour growth and metastasis. TF is overexpressed in several solid tumours, ${ }^{14}$ including in gynaecological cancers such as CC (100\%), ${ }^{15}$ endometrial cancer $14-100 \%)^{16}$ and ovarian cancer $(75-100 \%),{ }^{17}$ where it is associated with poor prognosis. ${ }^{18}$

Owing to its aberrant expression on tumour cells, TF is a target for ADC therapy. After binding with TF, TV releases its microtubule-disrupting agent MMAE inside the cell, causing cell cycle arrest and apoptotic cell death. 19,20 Moreover, as reported in preclinical studies, MMAE can diffuse across the cell membranes of neighbouring tumours, where it performs the same cytotoxic process.

In addition to its direct cell death activity, the cytotoxic effect of TV is also fulfilled through an indirect mechanism. The drug can activate the immune system through the FcyRlll expressed on natural killer cells, activating antibody-dependent cellular cytotoxicity (ADCC) and inducing immunogenic cell death; this process also results in the release of neoantigens, which play an important role in the specific adaptative immune response. ${ }^{21}$

TV was selected from a panel of six human TF-specific antibodies (HuMab-TF) because of its reduced interference with the pro-coagulant activity of TF. ${ }^{20}$

\section{Tisotumab vedotin in cervical cancer}

\section{Preclinical evidence}

The interaction between TF and ADC was firstly evaluated in preclinical studies. Breij, et al. studied the activity of monoclonal antibodies conjugated with cytotoxic agents directed against TF both in vitro and in vivo. ${ }^{20}$

Human tumour cells from pancreatic (ASPC-1, BXPC-3 and HPAF-II), colorectal (HCT-116), breast (MDA-MB-231), ovarian (SK-OV-3 and TOV21G) carcinoma, and epidermal adenocarcinoma (A431) were collected and tested for TF expression. QIFIKIT ${ }^{\circledR}$ (Dako, Glostrup, Denmark) analysis was performed using a mouse-derived antibody. In addition, a codonoptimized construct was generated for TF expression and transferred into HEK-293F cells or NSO cells. Human IgG1K TF antibodies (HuMab) were created through the immunization of HuMab mice. Three types of monoclonal antibodies (TF-011, TF-098 and TF-111) were conjugated with cytotoxic agents MMAE and MMAF. A protease-cleavable valine citrulline dipeptide was used for MMAE linking, whereas a non-cleavable maleimidocaproyl-containing linker was necessary for MMAF. The final result was an $\mathrm{ADC}{ }^{20}$

The binding between the ADC and TF was evaluated with flow cytometry. A negative interaction between HuMab-TF and physiological coagulation was also excluded: citrated human blood from healthy volunteers was incubated with humanized immunoglobulins, and thromboelastography was performed. The abovementioned cells were seeded and then incubated with monoclonal antibodies to evaluate HuMab-TF cytotoxicity in vitro. The percentage of dead cells relative to positive (100\% cell death) and negative (untreated cells) controls was measured. In vivo, xenograft models derived from the tumour cell line were injected into severe combined immunodeficient mice to investigate HuMab-TF. ${ }^{20}$

In vitro, the TF ADC showed high cytotoxicity against A431 and HPAFII cells, which presented high TF levels. In contrast, the TF ADC had a limited effect in cells with low TF expression, and no activity in the negative cells, suggesting that in vitro HuMab-TF activity was dependent on TF expression. ${ }^{20}$ 
Table 1: Published clinical trials

\begin{tabular}{|c|c|c|}
\hline & Hong, et al. ${ }^{24}$ & Coleman, et al. ${ }^{25}$ \\
\hline Phase & $\mid \mathrm{b} / \|$ & $\|$ \\
\hline Drug & Tisotumab vedotin & Tisotumab vedotin \\
\hline Setting & $\begin{array}{l}\text { Patients with relapsed, advanced and/or metastatic CC in whom } \\
\text { available standard treatments failed or who are not candidates } \\
\text { for standard therapy (innovaTV 201) }\end{array}$ & $\begin{array}{l}\text { Patients with relapsed or metastatic CC in whom available } \\
\text { standard treatments failed or who are not candidates for } \\
\text { standard therapy (innovaTV 204/GOG-3023/ENGOT-cx6) }\end{array}$ \\
\hline Patients enrolled, $\mathrm{n}$ & 55 & 101 \\
\hline Median age (range), years & $46(21-73)$ & $50(43-58)$ \\
\hline Median PFS $(95 \% \mathrm{Cl})$, months & $4.2(2.1-5.3)$ & $4.2(3.0-4.4)$ \\
\hline OS $(95 \% \mathrm{Cl})$, months & - & $12.1(9.6-13.9)$ \\
\hline ORR, \% & 24 & 24 \\
\hline Best response & PR (IRC assessed: CR) & CR \\
\hline
\end{tabular}

$C C=$ cervical cancer; $C l=$ confidence interval; $C R=$ complete response; $D O R=$ median duration of response; $I R C=I$ Independent Review Committee; $O R R=$ objective response rate; $O S=$ overall survival; $P F S=$ progression-free survival; $P R=$ partial response.

In vivo, the ADC containing MMAE demonstrated significant cytotoxic activity. Complete responses were observed in all mice treated with TF-011-MMAE and in most mice treated with TF-098-MMAE and TF-111MMAE. The responses were maintained after 20-30 days following the last treatment dose, and at recurrence. No response in the presence of low TF expression was observed in xenograft models. ${ }^{20}$

Patient-derived xenografts (PDX) probably represent the best model to study heterogeneous solid tumours and are particularly helpful for evaluating the activity of TF-targeting ADCs because of nonhomogeneous TF expression on the cell surface. In one study, seven PDX models were selected according to TF level of expression; in particular, in CC PDX models, TF positivity ranged from $25 \%$ to $50 \%$. PDX models were implanted into nude mice treated with TF-011-MMAE. Surprisingly, the CC PDX models showed tumour regression despite low TF expression. This result was likely due to the capacity of MMAE to diffuse across cell membranes and act on neighbouring TF-negative cells. ${ }^{22}$

\section{Clinical evidence}

Two clinical trials have been published addressing the role of TV in CC (Table 1). ${ }^{23-25}$ The innovaTV 201 study was a phase Ib/II trial with a doseescalation phase followed by a dose-expansion phase in patients with advanced or metastatic tumours who relapsed after or were not eligible for the standard of care. ${ }^{23,24}$ The expansion phase included patients with cervical, ovarian, endometrial, prostate, bladder, oesophageal or nonsmall-cell lung cancer. Eligible patients in the Cc cohort had measurable disease and had received at least four prior lines of chemotherapy. Patients with coagulation defects, ongoing major bleeding or residual grade 2 neuropathy were excluded. TF expression ${ }^{3}(1 \%)$ was confirmed in the majority of evaluable patients $(100 \%$ had membrane expression and $95 \%$ had cytoplasmatic expression); however, TF expression was not an inclusion criterion. A total of 55 patients were enrolled and received TV $2 \mathrm{mg} / \mathrm{kg}$ every 3 weeks for four cycles. In cases of stable disease, partial or complete response, patients could continue treatment up to 12 cycles and beyond if clinical benefit was still present (NCT03245736).

Safety was the primary endpoint. Patients received a prophylactic ocular medication with lubricating, vasoconstrictor and steroid eye drops to mitigate the risk of ocular adverse events (AEs). Overall, $56 \%$ of patients developed a grade 3 or worse $\mathrm{AE}$; the most common were anaemia
(11\%), fatigue (9\%) and vomiting (7\%). All patients developed at least one AE of any grade; the most common were fatigue (51\%), epistaxis (51\%), nausea (49\%) and conjunctivitis (42\%). Among AEs of special interest (AESI), the most common were ocular events, although 10 patients (9\%) discontinued treatment for peripheral neuropathy. Prophylactic medications significantly reduced the incidence of ocular events. ${ }^{22,23}$

Two fatal events occurred, both due to disease progression. Evaluation of anti-tumour activity was investigated as a secondary endpoint: the median progression-free survival (mPFS) was 4.2 months ( $95 \% \mathrm{Cl} 2.1-5.3$ months); the investigator-evaluated objective response rate (ORR) was $24 \%$ (95\% Cl 13-37\%) and was confirmed by the independent review committee (IRC) evaluation (ORR 22\%). ${ }^{22,23}$

Recently, Coleman, et al. evaluated the anti-tumour activity and safety of TV administered at a dosage of $2 \mathrm{mg} / \mathrm{kg}$ every 3 weeks in patients with advanced CC who progressed after no more than two prior treatment lines. ${ }^{25}$ The primary endpoint was ORR, and secondary endpoints included safety, tolerability, PFS, OS, time to response and duration of response (DOR). The correlation between TF expression and ORR was a prespecified exploratory endpoint. Among 102 screened patients, 101 were enrolled. At a median follow-up of 10 months, four patients were still on treatment and 33 were in follow-up, with a median duration of treatment of 4.2 months and a median of 6 cycles/patient. The IRC-confirmed ORR was $24 \%$ (95\% Cl 16-33\%) with seven complete responses (7\%) and 17 partial responses (17\%); disease control rate (DCR) was $72 \%$ (95\% Cl 63-81\%) with a median time to response of 1.4 months ( $95 \% \mathrm{Cl} 1.3-1.5$ months). Kaplan-Meier curves showed a mPFS of 4.2 months (95\% Cl 3.0-4.4 months) and a mOS of 12.1 months ( $95 \%$ Cl 9.6-13.9 months). In the exploratory analysis, no correlation between TF membrane expression and response to TV was found. ${ }^{25}$

Safety was consistent with previously reported AES, and treatmentrelated AEs occurred in 92 patients (93\%). Grade 3 or worse AEs were observed in 28 patients (28\%); most common were neutropenia (3\%), fatigue (2\%), ulcerative keratitis (2\%) and peripheral neuropathies (2\%). One grade 5 event was deemed related to treatment. ${ }^{25}$

According to the protocol, ocular, bleeding and neurological treatmentrelated AEs led to dose modification: $24 \%$ of patients had their dose interrupted, $22 \%$ had their dose reduced and $12 \%$ had their treatment 
Table 2: Ongoing trials in metastatic/recurrent cervical cancer

\begin{tabular}{|c|c|c|c|c|c|}
\hline Phase & Description & Drug & Primary endpoint & Status & Trial identifier \\
\hline $\mid \mathrm{b} / \mathrm{II}$ & $\begin{array}{l}\text { Safety and efficacy of TV monotherapy and in combination with } \\
\text { other cancer agents in patients with CC (ENGOT-CX8/GCT1015-05) }\end{array}$ & $\begin{array}{l}\text { TV } \\
\text { Bevacizumab } \\
\text { Pembrolizumab } \\
\text { Carboplatin }\end{array}$ & $\begin{array}{l}\text { DLT } \\
\text { ORR }\end{array}$ & Closed & NCT0378608126 \\
\hline |/II & $\begin{array}{l}\text { A trial of TV in Japanese patients with advanced solid malignancies } \\
\text { (innovaTV 206) }\end{array}$ & TV & $\begin{array}{l}\text { Safety } \\
\text { DLT } \\
\text { MTD } \\
\text { PK }\end{array}$ & $\begin{array}{l}\text { Active, not } \\
\text { recruiting }\end{array}$ & NCT0391374127 \\
\hline |/II & $\begin{array}{l}\text { Tolerability of TV dosed three times every } 4 \text { weeks ( } 3 q 4 w k) \text { in a } \\
\text { mixed population of patients with specified solid tumours }\end{array}$ & TV & Safety & Completed & NCT0255212128 \\
\hline$\|$ & TV in previously treated, recurrent or metastatic CC & TV & ORR & Active, not recruiting & NCT0343839629 \\
\hline III & $\begin{array}{l}\text { TV versus chemotherapy in recurrent or metastatic CC (innovaTV } \\
\text { 301) }\end{array}$ & $\begin{array}{l}\text { TV } \\
\text { Topotecan } \\
\text { Vinorelbine } \\
\text { Gemcitabine } \\
\text { Irinotecan } \\
\text { Pemetrexed }\end{array}$ & OS & Active, recruiting & NCT04697628 30 \\
\hline
\end{tabular}

$T V=$ tisotumab vedotin; $C C=$ cervical cancer; $D L T=$ dose-limiting toxicity; ORR = objective response rate; $M D T=$ maximum tolerated dose; $P K=$ pharmacokinetics; OS = overall survival.

discontinued. Among ocular AEs, the most common were conjunctivitis (23\%), dry eye (23\%) and keratitis (11\%). Only two patients experienced a grade $3 \mathrm{AE}$ and most of these events (86\%) had completely resolved 30 days after the last dose. 25

Bleeding AEs occurred in 39 patients (39\%), with only two (2\%) of grade 3. The most common bleeding events were epistaxis (30\%), vaginal haemorrhage $(7 \%)$ and haematuria (3\%). More than $90 \%$ of the events had resolved by the 30-day follow-up visit.. ${ }^{25} \mathrm{~A}$ total of 33 patients (33\%) experienced a peripheral neuropathy treatment-related $A E$, with seven events $(7 \%)$ of grade 3 . Unlike previously reported AESIS, neuronal damage was persistent in 90 patients (79\%) 30 days after the last dose of TV. Overall, $13 \%$ of patients experienced serious adverse events (SAES), the most common of which was sensor-motor neuropathy (2\%). ${ }^{25}$

\section{Ongoing trials}

Several phase I-III clinical trials are ongoing to evaluate the safety and efficacy of TV when administered alone or in combination with other drugs (Table 2). ${ }^{26-30}$ NCT0378608126 is a phase Ib/II open-label trial, investigating TV as monotherapy and in combination with bevacizumab, pembrolizumab or carboplatin in patients with recurrent or metastatic (stage IVB) CC. The trial consists of a dose-escalation phase and a dose-expansion phase. The dose-escalation phase will include patients with CC who have progressed during or after standard therapy and are ineligible for further standard treatments. This phase will be conducted to establish the maximum tolerated dose (MTD) and the recommended phase II dose (RP2D) of TV used in combination with bevacizumab (Arm A), pembrolizumab (Arm B) or carboplatin (Arm C). In the dose-expansion phase, patients who have not received prior systemic therapy will receive TV in combination with carboplatin (Arm D) or pembrolizumab (Arm E), while patients who have progressed on or after standard-of-care treatment will receive TV in combination with pembrolizumab (Arm F) or TV alone in a weekly schedule (Arm G). The primary endpoint of the expansion phase is ORR based upon the response evaluation criteria in solid tumours (RECIST) 1.1.

The innovaTV 206 study (NCT03913741)27 is a phase I/II, single-arm, open-label trial assessing the activity of TV in solid tumours, including $\mathrm{CC}$, in Japanese patients. The study comprises two parts: part 1 aims to evaluate MTD, RP2D and the safety profile of TV in patients with several solid malignancies; part 2 will include only patients with CC who have experienced disease progression after at least two previous chemotherapy lines, and will provide data regarding safety, tolerability, pharmacokinetics and anti-tumour activity.

Another phase I/II study (NCT02552121)28 established the tolerability of TV in a mixed population of patients with solid tumours, including ovarian, cervical, endometrial, bladder, prostate (castration resistant), oesophageal and non-small-cell lung cancer. The study included a dose-escalation phase, with increasing doses of TV in 28-day treatment cycles, followed by an expansion phase to explore the RP2D of TV as determined in the first part. Safety (AES, SAES and infusion-related AES) was the primary endpoint. A total of 33 patients were enrolled from November 2015 to December 2017 in two cohorts with different doses of TV $(0.9 \mathrm{mg} / \mathrm{kg}$ and $1.2 \mathrm{mg} / \mathrm{kg})$, both administered weekly three times every 4 weeks (3q4wk). Part 2 included only patients with cervical or ovarian cancer, and consisted of six cohorts designed to compare the weekly 3q4wk schedule (dose $1.2 \mathrm{mg} / \mathrm{kg}$ as defined in phase I trial) with the 3 -weekly $2.0 \mathrm{mg} / \mathrm{kg}$ schedule (1q3wk). Two cohorts were added because of severe ocular toxicity experienced by some patients on the 3q4wk schedule; affected patients switched from the 3q4wk schedule to $1 \mathrm{q} 3 \mathrm{wk}$ administration.

NCT03438396 ${ }^{29}$ is a single-arm, multicentre, phase $\|$ study of TV in patients with previously treated, recurrent or metastatic CC. All patients will receive TV $2.0 \mathrm{mg} / \mathrm{kg}$ intravenously 1q3wk until disease progression or toxicity. The primary endpoint will be the ORR evaluated according to RECIST 1.1 and assessed by IRC.

The innovaTV 301 study (NCT04697628)30 is a randomized, openlabel, phase III trial evaluating the efficacy of TV versus physician's chemotherapy choice in recurrent or metastatic CC. According to the study design, 482 participants will be randomized to receive TV $2.0 \mathrm{mg} / \mathrm{kg}$ 1q3wk versus standard chemotherapy including topotecan, vinorelbine, gemcitabine or irinotecan and pemetrexed according to investigators' choice. The primary endpoint is OS, and secondary endpoints are PFS, ORR, DOR, time to response and quality of life. 


\section{Discussion}

Advanced or recurrent CC progressing on first-line platinum-based chemotherapy still represents an urgent unmet clinical need, with few effective chemotherapy agents available. Besides immunotherapy, no other promising drugs are in clinical development.

The ADC TV could represent a concrete and promising option in the second-line setting in patients who do not respond to platinum firstline treatment. The $24 \%$ ORR reported in the CX6 study ${ }^{25}$ appears particularly interesting compared with historical chemotherapy activity data; furthermore, the characteristics of the patients enrolled in this trial are very close to those of the real-world advanced CC population, where patients are mostly pretreated with bevacizumab, which seems not to affect TV efficacy or to increase its toxicity. Of note, responses were detected in both squamous and adenocarcinoma histotypes, the latter of which is apparently less sensitive to immunotherapy, and TV may therefore represent a valuable alternative for these tumours. The toxicity profile of TV in terms of ocular events and bleeding seems manageable with the support of mitigation strategies, particularly those implemented for ocular events. The management of neurotoxicity requires caution, experience and careful follow-up, as neuropathy is the only long-lasting AESI reported in early clinical trials. The combination with carboplatin, bevacizumab and pembrolizumab, if feasible and active, could represent an alternative to the standard carboplatin-paclitaxel regimen, and warrants further exploration in comparison with the standard of care.
1. The Global Cancer Observatory. International Agency for Research on Cancer, EUCAN Cancer Factsheets, January, 2021. Available at: https://gco.iarc.fr/today/data/factsheets/ cancers/23-Cervix-uteri-fact-sheet.pdf (accessed 4 October 2021)

2. National Cancer Institute. 2021 SEER Cancer Statistics Review 2011-2017: Cancer of the Cervix Uteri. Available at: https:// seer.cancer.gov/statfacts/html/cervix.html (accessed 4 October 2021).

3. Tewari KS, Sill MW, Penson RT, et al. Bevacizumab for advanced cervical cancer: final overall survival and adverse event analysis of a randomised, controlled, open-label, phase 3 trial (Gynecologic Oncology Group 240). Lancet. 2017;390:1654-63.

4. Marth C, Landoni F, Mahner S, et al. Cervical cancer: ESMO clinical practice guidelines for diagnosis, treatment and followclinical practice guidelines for diagn
up. Ann Oncol. 2017;28:iv72-83.

5. Boussios $\mathrm{S}$, Seraj E, Zarkavelis G, et al. Management of patients with recurrent/advanced cervical cancer beyond first line platinum regimens: where do we stand? A literature review. Crit Rev Oncol Hematol. 2016;108:164-74.

6. Marabelle A, Le DT, Ascierto PA, et al. Efficacy of pembrolizumab in patients with noncolorectal high microsatellite instability/mismatch repair-deficient cancer: results from the phase II KEYNOTE-158 study. J Clin Onco. 2020;38:1-10

7. Lee EK, Liu JF. Antibody-drug conjugates in gynecologic malignancies. Gynecol Oncol. 2019;153:694-702.

8. Sun X, Ponte JF, Yoder NC, et al. Effects of drug-antibody ratio on pharmacokinetics, biodistribution, efficacy, and tolerability of antibody-maytansinoid conjugates. Bioconjug Chem. 2017:28:1371-81.

9. Beck A, Reichert JM. Antibody-drug conjugates: present and future. MAbs. 2014;6:15-7.

10. Birrer MJ, Moore KN, Betella I, Bates RC. Antibody-drug conjugate-based therapeutics: state of the science. J Nat Cancer Inst. 2019;111:538-49.

11. Von Minckwitz G, Huang C-S, Mano MS, et al. Trastuzumab emtansine for residual invasive HER2-positive breast cancer. $N$ Engl J Med. 2019;380:617-28.
12. Chu Al Tissue factor, blood coagulation and beyond: an overview. Int I Inflam. 2011;2011:367284.

3. Drake TA, Morrissey JH, Edgington TS. Selective cellula expression of tissue factor in human tissues. Implication for disorders of hemostasis and thrombosis. Am J Pathol 1989;134:1087-97.

14. Kasthuri RS, Taubman MB, Mackman N. Role of tissue factor in cancer. J Clin Oncol. 2009;27:4834-8.

5. Cocco E, Varughese J, Buza N, et al. Expression of tissue factor in adenocarcinoma and squamous cell carcinoma of the uterine cervix: implications for immunotherapy with hl-con1, a factor VII-IgGFC chimeric protein targeting tissue factor. BMC Cancer. 2011;11:263.

16. COCCO E, Hu Z, Richter CE, et al. hl-con1, a factor VII-IgGFC chimeric protein targeting tissue factor for immunotherapy of uterine serous papillary carcinoma. Br J Cancer. 2010:103:812 9.

17. Cocco $E$, Varughese J, Buza $N$, et al. Tissue factor expression in ovarian cancer: implications for immunotherapy with hl-con1, a factor VII-IgGF(c) chimeric protein targeting tissue factor. Clin Exp Metastasis. 2011;28:689-700

18. Förster Y, Meye A, Albrecht S, Schwenzer B. Tissue factor and tumor: clinical and laboratory aspects. Clin Chim Acta. 2006;364:12-21.

19. de Goeij BE, Lambert JM. New developments for antibody-drug conjugate-based therapeutic approaches. Curr Opin Immunol. 2016;40:14-23.

20. Breij ECW, de Goeij BECG, Verploegen S, et al. An antibody-drug conjugate that targets tissue factor exhibits potent therapeutic activity against a broad range of solid tumors. Cancer Res. 2014;74:1214-26.

21. Seidel UJE, Schlegel P, Lang P. Natural killer cell mediated antibody-dependent cellular cytotoxicity in tumor immunotherapy with therapeutic antibodies. Front Immuno 2013;4:76

22. Okeley NM, Miyamoto JB, Zhang X, et al. Intracellular activation of SGN-35, a potent anti-CD30 antibody-drug conjugate. Clin Cancer Res. 2010;16:888-97.

23. de Bono JS, Concin N, Hong DS, et al. Tisotumab vedotin in pationts with advanced or metastatic solid tumours (InnovaTV 201): a first-in-human, multicentre phase 1-2 trial. Lancet oncol. 2019;20:383-93.

24. Hong DS, Concin N, Vergote I, et al. Tisotumab vedotin in previously treated recurrent or metastatic cervical cancer. Clin Cancer Res. 2020;26:1220-8.

25. Coleman RL, Lorusso D, Gennigens C, et al. Efficacy and safety of tisotumab vedotin in previously treated recurrent or metastatic cervical cancer (innovaTV 204/GOG-3023/ENGOTcx6): a multicentre, open-label, single-arm, phase 2 study. Lancet Oncol. 2021;22:609-19.

26. ClinicalTrials.gov. Safety and Efficacy of Tisotumab Vedotin Monotherapy \& in Combination with Other Cancer Agents NCT03786081. Available at: https://clinicaltrials gov/ct2/show/ NCT03786081 (accessed 25 July 2021).

27. ClinicalTrials.gov. Open Label Phase $1 / 2$ Trial of Tisotumab Vedotin in Japanese Subjects with Advanced Solid Malignancies. ClinicalTrials.gov Identifier: NCT03913741. Available at: https://clinicaltrials.gov/ct2/show/study/ NCT03913741 (accessed 22 July 2021).

28. ClinicalTrials.gov. Dose-Escalating and Cohort Expansion Safety Trial of Tissue Factor Specific Antibody Drug Conjugate Tisotumab Vedotin (HuMax ${ }^{\circledR}$-TF-ADC) in Patients with Locally Advanced and/or Metastatic Solid Tumors Known to Express Tissue Factor. ClinicalTrials.gov Identifier: NCT02552121. Available at: https://clinicaltrials.gov/ct2/show/study/ NCT02552121 (accessed 22 July 2021).

29. ClinicalTrials.gov. A Single Arm, Multicenter, International Trial of Tisotumab Vedotin (HuMax@-TF-ADC) in Previously Treated Recurrent or Metastatic Cervical Cancer. ClinicalTrials.gov Identifier: NCT03438396. Available at: https://clinicaltrials.gov/ identifier: NCT03438396. Avaliable at: https.//clinicalt

30. ClinicalTrials.gov. A Randomized, Open-Label, Phase 3 Trial of Tisotumab Vedotin vs Investigator's Choice Chemotherapy in Second- or Third-Line Recurrent or Metastatic Cervical Cancer. ClinicalTrials.gov Identifier: NCT04697628. Available at: https:// clinicaltrials.gov/ct2/show/study/NCT04697628 (accessed 22 July 2021). 